\section{A E E T}

ASOCIACIÓN ESPAÑOLA DE ECOLOGÍA TERRESTRE

\title{
La aplicación del análisis de los anillos de crecimiento a interrogantes ecológicos: Un breve repaso de la Dendroecología en Hispanoamérica
}

\author{
M.M. Amoroso ${ }^{1,2, *}$, M.L. Suárez ${ }^{3}$ \\ (1) Consejo Nacional de Investigaciones Científicas y Técnicas, CCT Patagonia Norte, Av. De Los Pioneros 2350, 8400, San Carlos de Bariloche, Río \\ Negro, Argentina. \\ (2) Universidad Nacional de Río Negro, Escuela de Producción, Tecnología y Medio ambiente, Onelli 3076, 8430, El Bolsón, Río Negro, Argentina. \\ (3) Laboratorio Ecotono, INIBIOMA-CONICET, Universidad Nacional del Comahue, Quintral 1250, 8400, San Carlos de Bariloche, Río Negro, Argentina.
}

* Autor de correspondencia: M.M. Amoroso [mariano.amoroso@gmail.com]

> Recibido el 06 de julio de 2015 - Aceptado el 10 de julio de 2015

Amoroso, M.M., Suárez, M.A. 2015. La aplicación del análisis de los anillos de crecimiento a interrogantes ecológicos: Un breve repaso de la Dendroecología en Hispanoamérica. Ecosistemas 24(2): 1-6. Doi.: 10.7818/ECOS.2015.24-2.01

\section{La Dendrocronología}

La dendrocronología es la ciencia que utiliza la datación anual de los anillos de crecimiento de las plantas arbóreas y arbustivas leñosas para analizar los patrones temporales y espaciales de los procesos en las ciencias físicas y culturales. A pesar que el estudio de los anillos de crecimiento data del siglo XIX, se le atribuye a A. E. Douglass, fundador del Laboratory of Tree-Ring Research en la Universidad de Arizona, el desarrollo de la dendrocronología como ciencia. Dependiendo del uso y la aplicación de la serie de anillos, la dendrocronología reconoce diferentes subdisciplinas que varían según los autores. Entre ellas se encuentran la dendroarqueología, la dendroclimatología, la dendroecología y la dendrogeomorfología.

La dendroecología, en su definición más simple, utiliza las series de anillos datadas para responder a interrogantes ecológicos. Los anillos de crecimiento almacenan información acerca de los factores ambientales que afectan al crecimiento del árbol. Por ejemplo, las variaciones en la tasa de crecimiento de los árboles pueden indicar variaciones en las condiciones climáticas pasadas, y eventos discretos como la ocurrencia de incendios o avalanchas de nieve, quedan también registradas al dejar marcas visibles conocidas comúnmente como cicatrices. Los datos de resolución anual obtenidos por el correcto datado dendrocronológico nos facilita el análisis de muchos procesos ecológicos a amplias escalas temporales que no podría investigarse con los datos obtenidos periódicamente. El monitoreo a largo plazo de parcelas permanentes no siempre se encuentra disponible, o es apenas incipiente en muchas regiones. Por ello, cuando no se cuenta con parcelas permanentes o éstas son muy recientes, y si las especies bajo estudio cuentan con formación anual de anillos de crecimiento, la utilización de métodos dendroecológicos permite obtener importante información acerca del sistema bajo estudio.
Diversos estudios ecológicos en todo el mundo se han beneficiado de la información almacenada en los anillos de crecimiento. En Hispanoamérica, son numerosos los estudios que utilizan enfoques dendroecológicos para reconstruir los regímenes de disturbio de diferentes tipos de vegetación, así como para identificar patrones de crecimiento resultante de cambios ambientales. De este modo, la dendroecología ha sido utilizada para identificar frecuencia de ocurrencia de disturbios (ej. incendios, ataques de insectos, terremotos, vulcanismo, eventos climáticos extremos) y su influencia sobre la estructura y dinámica de los sistemas estudiados. Asimismo, enfoques dendroecológicos se han utilizado para caracterizar los patrones de crecimiento de los árboles y estudiar aspectos importantes del decaimiento forestal, tema de creciente interés en el contexto del cambio climático. Por último, la utilización de anillos de crecimiento, ha sido de vital importancia en estudios cuyos objetivos involucran a la gestión forestal, permitiendo determinar entre otras cosas, turnos de corta y aprovechamiento.

Este trabajo recopila una muestra muy diversa de la investigación contemporánea en Hispanoamérica sobre la utilización de series de anillos de especies leñosas para responder a interrogantes ecológicos en diferentes ecosistemas. No se pretendió realizar una revisión completa, pero sí proporcionar al menos una muestra de las principales aplicaciones y su amplia utilidad para el estudio de diversos procesos ecológicos en un amplio espectro de ecosistemas, además de contextualizar las ocho contribuciones realizadas en el presente monográfico.

\section{Incendios}

Uno de los grandes disturbios modeladores del paisaje ha sido el fuego, sea éste de origen natural (rayos) como estrechamente ligado a las actividades humanas (expansión de la frontera agrícola-ganadera, uso de la tierra, etc.). En este contexto, conocer los regímenes de fuego pasados, su variabilidad natural, su influencia 
como agente estructurador de las comunidades, y como el hombre ha influenciado en modificar los regímenes de fuego ha sido de gran interés para la ecología. En particular, la dendroecología del fuego ha aportado al estudio de la determinación de los regímenes de incendios a fin de comprender las consecuencias sobre la estructura y dinámica de los sistemas donde ocurren en una amplia escala temporal. El estudio del régimen de fuego implica la caracterización de la frecuencia, intensidad, extensión y estacionalidad de la ocurrencia de fuegos en las comunidades donde ocurren. Para ello, la dendroecología utiliza datos aportados por anillos de crecimiento (fechado de inicio de rodales, variabilidad en los patrones de crecimiento), y los que surgen de la datación de cicatrices de fuego presentes en individuos que han resultado afectados por el fuego, para aproximar a los patrones y procesos relacionados a la ocurrencia de incendios.

En Hispanoamérica la utilización de enfoques dendroecológicos para el estudio del fuego ha sido amplia. El fechado de marcas de incendio en secciones transversales de diferentes especies de árboles ha permitido determinar la frecuencia y estacionalidad de fuegos pasados, así como la diferenciación entre fuegos de gran magnitud y fuegos de pequeña extensión (Medina et al. 2000, Fule et al. 2008, Cerano Paredes et al. 2010). Asimismo, la estimación de fechas de establecimiento de árboles, junto al fechado de cicatrices de incendios, ha permitido estudiar los efectos sinérgicos entre la ocurrencia de incendios y otros factores como clima (Grau y Veblen 2000, González y Veblen 2006, Mundo et al. 2013) y/o historias de manejo sobre las comunidades leñosas (Bogino et al. 2015). Por otra parte, el desarrollo de robustas cronologías de fuego, ha permitido determinar la manera en que la frecuencia de fuego refleja los cambios en las actividades humanas en un contexto histórico, así como la influencia de las condiciones físicas que impone el ambiente donde ocurren (Kitzberger et al. 1997, Veblen et al. 1999, González 2005, Veblen et al. 2009).

\section{Insectos}

En las últimas décadas, han aumentado los fenómenos de decaimiento y mortalidad de bosque asociado entre otros, a factores bióticos relacionados al cambio global (ej. insectos barrenadores y defoliadores). En particular, el reciente aumento de las temperaturas ha favorecido el aumento y expansión de poblaciones de insectos forestales, provocando daños en los bosques de todo el mundo (Anderegg et al. 2015). Este aumento episódico en la densidad de insectos forestales es considerado una perturbación importante en muchas comunidades boscosas, produciendo mortalidad de árboles o bien defoliación temporal o permanente del dosel. En algunas ocasiones, vastas áreas de bosques sufren mortalidad masiva durante el incremento poblacional de algún insecto, por lo que se provocan cambios en el paisaje, con importantes implicancias ecológicas y pérdidas económicas. En este campo, la dendroecología ha aportado al estudio del efecto del ataque de insectos en el crecimiento radial de los árboles, permitiendo además, reconstruir la frecuencia, intensidad y distribución espacial de los episodios de ataques.

Por ejemplo, en bosques de España y Argentina, algunos estudios han evaluado la efectividad del uso de técnicas dendroecológicas para detectar eventos pasados de defoliación, así como cuantificar la reducción radial derivada del ataque de lepidópteros (Camarero et al. 2003, Paritsis et al. 2009, Sangüesa-Barreda et al. 2014). Del mismo modo, la utilización de testigos radiales ha permitido caracterizar la pérdida de vigor en árboles, debido al ataque de insectos barrenadores o perforadores (ej. Coleópteros), los cuales son los principales agentes de mortalidad y/o decaimiento de algunos tipos de bosques, produciendo grandes pérdidas económicas (Ferrero et al 2013). Sin embargo, a diferencia de los insectos defoliadores, son pocos los estudios que relacionan directamente los patrones de crecimiento de los árboles y el ataque puntual de un insecto barrenador, abundando más los estudios que colocan al ataque de los coleópteros en el contexto de decaimiento forestal iniciado por algún factor estresante como la sequía.
Asimismo, la dinámica de las poblaciones de insectos se ve afectada por la variabilidad climática, ya que el ciclo de vida de los insectos puede acelerarse con el aumento en la temperatura (Hódar et al. 2003). En este sentido, la utilización de cronologías de ancho de anillos para reconstruir la historia de defoliaciones masivas, ha permitido relacionar la variabilidad en la dinámica de las poblaciones de insectos defoliadores con la variabilidad climática de la región (Paritsis y Veblen 2011, Hernández Alonso et al. 2005).

\section{Estructura y dinámica forestal (natural y como resultado de disturbios o agentes abióticos)}

En el campo de la ecología forestal, el fechado y la medición de la variación en el crecimiento de los árboles de un rodal ha sido de gran utilidad en el estudio de procesos ecológicos, los cuales tienden a ser lentos y por lo tanto indetectables sin la ayuda de técnicas retrospectivas (Kitzberger et al. 2000). En particular, si se requiere información acerca de la estructura de edades de un rodal, es preciso fechar con cierta precisión, los árboles del rodal estudiado. Mediante el estudio de los anillos de crecimiento, además de conocer la edad de los árboles, se puede saber, entre otras cosas, su tasa de crecimiento anual, y la capacidad de regeneración de la población. Luego, el análisis de la distribución de edades resultante, sumado a información acerca de los factores bióticos y abióticos que pueden ejercer influencia sobre el rodal en estudio, darán cuenta de la historia del sistema de estudio y de las características de las perturbaciones que pudieron haber afectado al mismo (Dussart et al. 1998, Villalba y Veblen 1997, Pollman 2003, Rozas 2003, 2004a, Brienen 2005).

Asimismo, un enfoque dendroecológico permite abordar el estudio de la dinámica del bosque. Mediante el fechado de establecimiento y mortalidad de árboles, el análisis de la variación en el crecimiento (liberaciones/supresiones), la observación de sincronía en las respuestas de los árboles del rodal, así como de los patrones obtenidos, se puede obtener una reconstrucción aproximada de la dinámica del sistema de estudio (Olano et al. 2008, Suárez y Kitzberger 2010, Dussart et al. 2011, Rozendaal et al. 2011, Bogino et al. 2015). Esta información, sumada a datos sobre factores bióticos y abióticos que pueden ejercer influencia sobre el rodal en estudio, permite analizar las características de los disturbios que pudieron haber modelado el mismo. De este modo, un enfoque dendroecológico no solo permite representar los patrones estructurales de un bosque, sino que además se puede inferir acerca de los procesos que han llevado a los mismos.

\section{Decaimiento del bosque}

El decaimiento del bosque es un proceso común a varios ecosistemas boscosos en el mundo que responde a la acción de diversos factores que interactúan de manera compleja. En este proceso el árbol comienza a perder vigor lentamente pudiendo ocurrir la muerte del individuo al cabo de varios años o décadas. Asimismo, y a diferencia de las perturbaciones tales como el fuego, los deslizamientos o las avalanchas, las causas que producen el decaimiento y la posterior muerte de los árboles no son fácilmente identificables, ni la muerte de los árboles sincrónica. Los patrones de crecimiento radial se han utilizado exitosamente para estudiar el declive en el crecimiento ya que se consideran buenos indicadores de las respuestas de los árboles a los factores de estrés a largo plazo. La mayoría de los estudios en Hispanoamérica se han centrado en los factores de estrés climáticos relacionados con el declive en crecimiento radial, sin embargo algunos han indagado en el efecto de factores bióticos o en los procesos involucrados en el declive de crecimiento, como también en la muerte final de los árboles y los cambios en la dinámica poblacional en bosque en decaimiento.

El decaimiento del bosque inducido por sequías extremas es común a diferentes tipos de bosques. Diferentes estudios retrospectivos han resaltado el rol de las sequías en el declive en el crecimiento radial de árboles y propuesto que las mismas actúan como un factor de predisposición (Mundo et al. 2010, SánchezSalguero et al. 2010, Linares et al. 2010, Amoroso et al. 2015, Camarero et al. 2015). 
En algunos procesos de decaimiento, el efecto de las sequías se ve potenciado o acompañado por la acción de factores bióticos como plantas hemiparásitas, hongos patógenos e insectos. Son particularmente importantes los estudios que han abordado como estos factores interactúan e inducen el declive en crecimiento radial (Sangüesa-Barreda et al. 2012, 2015a). De la misma manera, en otros procesos de decaimiento se ha documentado como el uso o manejo pasado del bosque puede aumentar la vulnerabilidad de estos al efecto de tanto con factores abióticos y bióticos como la sequía y los hongos patógenos (Camarero et al. 2011, SangüesaBarreda et al. 2015a).

Los patrones de crecimiento radial, en combinación con el estudio de otras variables del tipo fisiológicas, han permitido indagar en más detalle sobre los procesos o mecanismos involucrados en el declive de crecimiento y su relación con las sequías (Camarero et al. 2012, Linares y Camarero 2012, Sangüesa-Barreda et al. 2013, Voltas et al. 2013).

Los procesos de decaimiento desencadenan, eventualmente, la muerte de gran parte de los individuos de un rodal. Recientes estudios han destacado el rol del estrés hídrico producido por sequías determinando la mortalidad de árboles en bosque en decaimiento (Gea-Izquierdo et al. 2014, Amoroso et al. 2015).

Si bien en la mayoría de los casos estudiados los patrones de declive radial resultan como consecuencia de estos procesos de decaimiento, algunos estudios ponen de manifiesto el rol de la competencia por recursos entre individuos en el desarrollo de estos procesos (Amoroso et al. 2012a, 2015, Linares et al. 2010, Rodríguez-Catón et al. 2015)

Finalmente, cabe destacar que son escasos los estudios dendroecológicos de bosques en decaimiento que han abordado aspectos de la dinámica como ser los cambios en el establecimiento, los patrones de mortalidad y crecimiento radial (Amoroso y Larson 2010a, Amoroso et al. 2012b), debiendo estos aspectos ser estudiados en otros ecosistemas.

\section{Gestión forestal}

El uso de los anillos anuales de crecimiento de los árboles ha colaborado en diferentes aspectos con la gestión forestal. Esto incluye la determinación de las tasas de crecimiento radial para la determinación de turnos corta o rotaciones, la determinación de la productividad, la evaluación de prácticas silviculturales como los raleos, el impacto de prácticas y usos pasados de los bosques, y el estudio de procesos naturales para su aplicación en la gestión.

La información almacenada en los anillos de crecimiento de las especies leñosas permite determinar los ritmos de crecimiento diamétrico que a su vez pueden ser la base para establecer pautas de manejo sostenible. Son varios los estudios que han utilizado este enfoque tanto en bosques tropicales (Brienen y Zuidema 2006a,b, Schongart 2008, López et al. 2012, 2013) como en bosques templados (Chauchard y Sbrancia 2003, Villagra et al. 2005a,b) para, a partir de estos, determinar los turnos de corta o rotaciones biológicas. De manera similar, los incrementos en el ancho de los anillos han permitido analizar la productividad del bosques en términos de volumen (Cruz Johnson et al. 2007) o través de la estimación de los incrementos en biomasa (Worbes y Raschke 2012).

Son varios los trabajos que ha utilizado técnicas dendroecológicas para evaluar el impacto de prácticas y usos pasados de los bosques en el largo plazo. Particularmente en España y Chile, estos estudios han permitido estudiar como el crecimiento, estructura, composición y dinámica de las estructuras residuales de los mismos respondió a diferentes practicas forestales (Pollman 2002, Rozas 2003, 2004b, 2005, Olano et al. 2008, Rozas et al. 2008, Szejner et al. 2011). Estudios dendrocronológicos han demostrado ser también una valiosa herramienta para la validación objetiva del éxito de prácticas silviculturales en un corto plazo. Tal es el caso del estudio de la respuesta en crecimiento radial de raleos (MartínBenito et al. 2010, Pérez-de-Lis et al. 2011).
Finalmente, los estudios dendroecológicos de reconstrucción de disturbios pueden ser usados como una herramienta complementaria para diseñar sistemas silvícolas al permitirnos reconstruir patrones de establecimiento, crecimiento radial y mortalidad en el tiempo, y así realizar recomendaciones de manejo sobre la base de la dinámica poblacional (Amoroso y Larson 2010b, Amoroso 2013).

\section{Otras aplicaciones de la dendroecología}

Otros estudios ecológicos se han beneficiado de la información almacenada en los anillos de crecimiento de las especies leñosas. El uso de técnicas dendroecológicas ha permitido la reconstrucción y datación de diferentes tipos de perturbaciones. Para el caso de los eventos avalanchas de nieve, son pioneros los trabajos en los bosques de Nothofagus pumliio en los Andes patagónicos de Argentina que permitieron la reconstrucción temporal y espacial de este tipo de eventos (Mundo et al. 2007, Casteller et al. 2008, 2009, 2011). En estos mismos bosques, estas técnicas permitieron reconstruir la historia de caídas masivas de árboles ocasionadas por fuertes tormentas y comprender la influencia de esta perturbación en la dinámica de estos bosques (Rebertus et al. 1997). De manera similar, Paolini et al. (2005) utilizaron técnicas dendroecológicas para datar la fecha la ocurrencia de deslizamientos en el norte de Argentina. Asimismo, Kitzberger et al. (1995) examinaron la influencia de la variación climática y la estabilidad del sustrato sobre el efecto de los terremotos en el norte de la Patagonia, Argentina, utilizando un enfoque dendroecológico. En el caso del datado de avenidas torrenciales en torrentes españoles, además del enfoque dendroecológico para el datado de eventos, han sido de gran utilidad indicadores anatómicos del leño (Ballesteros-Canovas et al. 2010).

Estudios dendroecológicos han también permitido estudiar procesos relacionados a la formación de los anillos de crecimiento o de otros procesos que quedan registrados en los mismos durante su formación. En algunas especies puede llegar a producirse la muerte cambial en sectores del fuste de individuos vivos (Amoroso y Daniels 2010). En otros casos, se producen estructuras anatómicamente anómalas y ecofisiológicamente patológicas que resultan de la ocurrencia de eventos extremos en la temperatura como las de heladas (Hadad et al. 2012).

\section{Consideraciones finales}

El presente monográfico pretende resaltar parte de los aportes al campo de la dendroecología en Hispanoamérica. Los 8 trabajos que forman parte del mismo, 6 de investigación y 2 de revisión, dan cuenta de la diversa y activa investigación que se viene desarrollando en diferentes ecosistemas utilizando la información almacenada en los anillos de crecimiento. La contribución de Arenas-Castro et al. 2015, describe la composición y estructura demográfica de poblaciones de Pyrus bourgaeana (piruétano) en dos hábitats diferentes a través del estudio de la dinámica del crecimiento radial y la edad de los individuos. Al estudio de decaimiento forestal mediante un enfoque dendroecológico, se suma el presente en este monográfico donde Sangüesa-Barreda et al.(2015b), utilizando cuatro casos de estudio en bosques dominados por distintas especies de árboles afectados por diferentes estreses bióticos. Estos autores estudian el papel de diferentes factores bióticos y las sequías en el decaimiento diferentes ecosistemas boscosos. Con objetivos en gestión forestal, este monográfico aporta dos nuevas contribuciones. López y Villalba (2015) evalúan diferentes criterios de gestión forestal para 12 especies de bosques tropicales de Bolivia a través de métodos dendrocronológicos y los comparan con pautas de manejo establecidas para promover el manejo sostenible de los mismos. De manera similar, Tíscar (2015) propone un modelo de ciclo forestal para la gestión sostenible de los bosques de Pinus nigra deducido a partir del estudio del establecimiento y crecimiento de regenerado en claros. En el contexto de aplicaciones dendroecológicas al estudio de avenidas torrenciales, este mono- 
gráfico presenta el trabajo de García-Oteyza et al. 2015, donde se estudia el número y la distribución temporal de avenidas y flujos torrenciales a través de la datación de eventos de flujos torrenciales ocurridos en una cuenca en Lleida para utilizando técnicas dendrogeomorfológicas. Por último, Pompa-García y Domínguez-Calleros 2015 , en el presente monográfico, aportan al conocimiento de la variabilidad interanual en la formación del leño de Pinus cooperi en México al examinar el rol de las sequías en la formación de la madera temprana y tardía en estos.

El análisis conjunto de diferentes estudios dendroecológicos permite muchas veces un acabado y mejor entendimiento de ecosistemas boscosos. En este sentido, son de gran utilidad los trabajos de revisión como los que se presentan en este monográfico. Dussart et al. (2015) presentan una revisión del uso de los estudios dendroecológicos en bosques de caldén (Prosopis caldenia) en Argentina para relacionar estos estudios con la evolución del pensamiento científico y contexto socio-económico de estos bosques, para proyectar futuros desafíos de estos estudios en relación al manejo y la conservación de estos ecosistemas. Olano y Rozas (2015), por su parte, realizan una revisión bibliográfica para resaltar el uso de la dendrocronología en el estudio de diferentes aspectos relacionados a la funcionalidad de la sabina albar (Juniperus thurifera) y la dinámica de sus ecosistemas.

Esperamos que el presente monográfico despierte el interés de estudiantes e investigadores y contribuya a ampliarlos enfoques dendroecológicos en la investigación ecológica a través la aplicación de métodos dendroecológicos.

\section{Agradecimientos}

Como editores invitados del presente monográfico queremos agradecer a todos los autores por sus contribuciones y a todos los revisores por su ayuda en la revisión de los diferentes manuscritos. También queremos agradecer especialmente a Juan Blanco quien nos invitó a editar un número especial sobre un tema que nos apasiona, y a Juan, Luis Cayuela y Leyre Jiménez por su ayuda y labor de coordinación editorial.

\section{Referencias}

Amoroso, M.M. 2013. Recomendaciones de manejo para rodales de ciprés de la cordillera con sintomatología de "mal del ciprés": el estudio de la dinámica poblacional como línea de base. Actas del 4to Congreso Forestal Argentino y Latinoamericano, Iguazú 2013.

Amoroso, M.M., Larson, B.C. 2010a. Stand development patterns as a consequence of the mortality in Austrocedrus chilensis forests. Forest Ecology and Management 259: 1981-1992.

Amoroso, M.M., Larson, B.C. 2010b. Can a natural experiment be used as a tool to design partial cutting regimes? The decline of Austrocedrus chilensis forests, an example. Journal of Forest Research 15: 38-45.

Amoroso, M.M., Daniels, L.D. 2010. Cambial mortality in declining Austrocedrus chilensis forests: implication for stand dynamics studies. Canadian Journal of Forest Research 40: 885-893.

Amoroso, M.M., Daniels, L.D., Larson, B.C. 2012a. Temporal patterns of radial growth in declining Austrocedrus chilensis forests in Northern Patagonia: the use of tree-rings as an indicator of forest decline. Forest Ecology and Management 265: 62-70.

Amoroso, M.M., Suarez, M.L., Daniels, L.D. 2012b. Nothofagus dombey regeneration in declining Austrocedrus chilensis forests: effects of overstory mortality and climatic events. Dendrochronologia 30: 105-112.

Amoroso, M.M., Daniels, L.D., Villalba, R., Cherubini, P. 2015. Does drought incite tree decline and death in Austrocedrus chilensis forests? Journal of Vegetation Science DOI: 10.1111/jvs.12320.

Anderegg, W,R., Hicke, J.A., Fisher, R.A., Allen, C.D., Aukema, J., Bentz, B., et al. 2015.Tree mortality from drought, insects, and their interactions in a changing climate. New Phytologist DOI: 10.1111/nph.13477.

Arenas-Castro, S., Fernández-Haeger, J., Jordano-Barbudo, D. 2015. Estructura de edades, tamaños y crecimiento en una población local de piruétano (Córdoba, España). Ecosistemas 24(2): 7-14.
Ballesteros, J.A., Díez-Herrero, A., Bodoque, J.M., Stoffel, M., Bollschweiler, M., Hitz, O. 2006. Caracterización de la señal anatómica en la madera de árboles afectados por avenidas torrenciales. Trabajos de Geomorfología en España 191-194.

Ballesteros-Canovas, J.A., Stoffel, M., Bodoque del Pozo, J.M., Bollschweiler, M., Hitz, O.M., Diez-Herrero, A. 2010. Changes in wood anatomy in tree rings of Pinus pinaster Ait. following wounding by flash floods. TreeRing Research 66: 93-103

Bogino, S., Roa-Giménez, S.C., Velasco-Sastre, A.T., Cangiano, M.L., RisioAllione, L., Rozas, V. 2015. Synergetic effects of fire, climate, and management history on Prosopiscaldenia recruitment in the Argentinean pampas. Journal of Arid Environments 117:59-66.

Brienen, R.J.W. 2005. Tree rings in the tropics: a study on growth and ages of Bolivian rain forest trees. Utrecht University. Utrecht, Países Bajos.

Brienen, R.J.W., Zuidema, P.A. 2006a. Lifetime growth patterns and ages of Bolivian rain forest trees obtained by tree ring analysis. Ecology 94 : 481-493.

Brienen, R.J.W., Zuidema, P.A. 2006b. The use of tree rings in tropical forest management: Projecting timber yields of four Bolivian tree species. Forest Ecology and Management 226: 256-267.

Camarero, J.J., Martín, E., Gil-Pelegrín, E. 2003. The impact of a needleminer (Epinotia subsequana) outbreak on radial growth of silver fir (Abies alba) in the Aragón Pyrenees: a dendrochronological assessment. Dendrochronologia 21:3-12.

Camarero, J.J., Bigler, C., Linares, J.C., Gil-Pelegrín, E. 2011. Synergistic effects of past historical logging and drought on the decline of Pyrenean silver fir forests. Forest Ecology and Management 262: 759-769.

Camarero, J.J., Sangüesa Barreda, G., Alla, A.Q, González de Andrés, E., Maestro Martínez, M., Vicente-Serrano, S.V. 2012. Los precedentes y las respuestas de los árboles a sequías extremas revelan los procesos involucrados en el decaimiento de bosques mediterráneos de coníferas. Revista Ecosistemas 21: 22-30.

Camarero, J.J., Gazol, A., Sangüesa-Barreda, G., Oliva, J., VicenteSerrano, S.M. 2015. To die or not to die: early warnings of tree dieback in response to a severe drought. Journal of Ecology 103: 44-57.

Casteller, A., Christen, M., Villalba, R., Martínez, H., Stöckli, V., Leiva, J. C., Bartelt, P. 2008. Validating numerical simulations of snow avalanches using dendrochronology: The Cerro Ventana event in Northern Patagonia, Argentina. Natural Hazards and Earth Systems Sciences 8: 433-443.

Casteller, A., Villalba, R., Mayer, A., Stöckli, V. 2009. Reconstrucción espacial y temporal de la ocurrencia de avalanchas de nieve en los Andes patagónicos utilizando técnicas dendrocronológicas. Revista Chilena de Historia Natural 82: 245-264.

Casteller, A., Villalba, R., Araneo, D., Stöckli, V. 2011. Reconstructing temporal patterns of snow avalanches at Lago del Desierto, southern Patagonian Andes. Cold Regions Science and Technology 67: 68-78.

Cerano Paredes, J., Villanueva Díaz, J., Fulé, P. Z. 2010. Reconstrucción de incendios y su relación con el clima para la Reserva Cerro El Mohinora, Chihuahua. Revista mexicana de ciencias forestales 1:63-74.

Chauchard, L., Srancia, R. 2003. Modelos de crecimiento diamétrico para Nothofagus obliqua. Bosque 24: 3-16.

Cruz Johnson, P., HoneymanLucchini, P., Pezo Correa, A., Schulze del Canto, C. 2007. Análisis de crecimiento de árboles maduros de lenga (Nothofagus pumilio) en bosques de la XII Región, Chile. Bosque 28: 18-24.

Dussart, E., Lerner, P., Peinetti, R. 1998. Long term dynamics of 2 populations of Prosopis caldenia Burkart. Journal of Range Management 51: 685-691.

Dussart, E.G., Chirino, C.C., Morici, E.A., Peinetti, R.H. 2011. Reconstrucción del paisaje del caldenal pampeano en los últimos 250 años. Quebracho (Santiago del Estero) 19:54-65.

Dussart, E., Medina, A., Bogino, S. 2015. Dendroecología en la pampa Argentina: investigaciones actuales, pasadas y futuros desafíos. Ecosistemas 24(2): 51-59.

Ferrero, M.E., Coirini, R.O., Díaz, M.P. 2013. The effect of wood-boring beetles on the radial growth of Prosopis flexuosa DC. in the arid Chaco of Argentina. Journal of Arid Environments 88:141-146.

Fulé, P.Z., Ribas, M., Gutiérrez, E., Vallejo, R., Kaye, M.W. 2008. Forest structure and fire history in an old Pinusnigra forest, eastern Spain. Forest Ecology and Management 255: 1234-1242. 
García-Oteyza, J., Génova, M., Calvet, J., Furdada, G., Guinau, M.,DíezHerrero, A. 2015. Datación de avenidas torrenciales y flujos de derrubios mediante metodologías dendrogeomorfológicas (barranco de Portainé, Lleida, España). Ecosistemas 24(2): 43-50.

Gea-Izquierdo, G., Guibal, F., Joffre, R., Ourcival, J-M., Simioni, G., Guiot, J. 2014. Drought induced decline could portend widespread pine mortality at the xeric ecotone in managed mediterranean pine-oak woodlands. Forest Ecology and Management 320: 70-82.

González, M.E. 2005. Fire history data as reference information in ecological restoration. Dendrochronologia 22: 149-154.

González, M.E., Veblen, T.T. 2006. Climatic influences on fire in Araucaria araucana-Nothofagus forests in the Andean cordillera of south-central Chile. Ecoscience 13:342-350.

Grau, R.H., Veblen, T.T. 2000. Rainfall variability, fire and vegetation dynamics in neotropical montane ecosystems in north-western Argentina. Journal of Biogeography 27: 1107-1121.

Hadad, M.A., Amoroso, M.M., Roig Juñent, F.A. 2012. Frost ring distribution in Araucaria araucana trees from the xeric forests of Patagonia, Argentina. Bosque 33: 309-312.

Hernández Alonso, R., Pérez Fortea, V., Camarero, J.J., Montoya, R., Sánchez Peña, G. 2005. Efectos de la defoliación inducida por la procesionaria del pino (Thaumetopoea pityocampa) sobre el crecimiento y la supervivencia de Pinus nigra e interacciones con el clima durante el periodo 1992-2004. Actas del IV Congreso Forestal Español, Zaragoza, España pp. 366-374.

Hódar, J.A., Castro, J. Zamora, R. 2003. Pine processionary caterpillar Thaumetopoea pityocampa as a new threat for relict Mediterranean Scots pine forests under climatic warming. Biological Conservation 110:123-129.

Kitzberger T., Veblen T.T., Villalba R. 1995. Tectonic influences on tree growth in northern Patagonia, Argentina: The roles of substrate stability and climatic variation. Candian Journal of Forest Research 25:1684-96

Kitzberger, T., Veblen, T.T., Villalba, R. 1997. Climatic influences on fire regimes along a rain forest-to-xeric woodland gradient in northern Patagonia, Argentina. Journal of Biogeography 24: 35-47.

Kitzberger, T., Veblen T. T. Villalba R. 2000. Métodos dendroecológicos y sus aplicaciones en estudios de dinámica de bosques templados de Sudamérica. En: F.A.R. (comp) (ed.). Dendrocronología en América Latina, pp. 17-78. EDIUNC, Mendoza. Argentina.

Linares J.C., Camarero, J.J., Bowker, M.A., Ochoa, V., Carreira, J.A. 2010. Stand-structural effects on Heterobasidion abietinum-related mortality following drought events in Abies pinsapo. Oecologia 164: 1107-1119.

Linares, J.C., Camarero, J.J. 2012. Growth patterns and sensitivity to climate predict silver fir decline in the Spanish Pyrenees. European Journal of Forest Research 131: 1001-1012.

López, L., Villalba, R. 2015. Criterios de gestión forestal para 12 especies de los Bosques Nativos Tropicales de Bolivia a través de métodos dendrocronológicos. Ecosistemas 24(2): 24-29.

Lopez, L., Villalba, R., Peña-Claros, M. 2012. Ritmos de crecimiento diamétrico en los bosques secos tropicales: aportes al manejo sostenible de los bosques de la provincia biogeográfica del Cerrado Boliviao. Bosque 33: 99-107.

Lopez, L., Villalba, R., Bravo, F. 2013. Cumulative diameter growth and biological rotation age for seven tree species in the Cerrado biogeographical province of Bolivia. Forest Ecology and Management 292: 49-55.

Martín-Benito, D., Del Río, M., Heinrich, I., Helle, G., Cangellas, I. 2010. Response of climate-growth relationships and water use efficiency to thinning in a Pinus nigra afforestation. Forest Ecology and Management 259: 967-975.

Medina, A., Dussart, E.G., Estelrich, H.D., Morici, E.A. 2000. Reconstrucción de la historia del fuego en un bosque de Prosopis caldenia (Burk.) de Arizona, sur de la provincia de San Luis. Multequina 9.

Mundo, I.A., Barrera, M.D., Roig, F.A. 2007. Testing the utility of Nothofagus pumilio for dating a snow avalanche in Tierra del Fuego, Argentina. Dendrochronologia 25: 19-28.

Mundo, I.A., El Mujtar, V.A., Perdomo, M.H., Gallo, L.A., Villalba, R., Barrera, M.D. 2010. Austrocedruschilensis growth decline in relation to drought events in Northern Patagonia, Argentina. Trees 24: 561-570.
Mundo, I. A., Wiegand, T., Kanagaraj, R., Kitzberger, T. 2013. Environmental drivers and spatial dependency in wildfire ignition patterns of northwestern Patagonia. Journal of environmental management 123 : 77-87.

Olano, J.M., Rozas, V. 2015. Desde el cámbium hasta el bosque: evaluando las respuestas de Juniperus thurifera L. a los condicionantes ambientales y antrópicos a múltiples escalas. Ecosistemas 24(2): 60-68.

Olano, J.M., Rozas, V., Bartolomé, D., Sanz, D. 2008. Effects of changes in traditional management on height and radial growth patterns in a Juniperus thurifera L. woodland. Forest Ecology and management 255: 506-512.

Paolini, L., Villalba, R. Grau, H.R. 2005. Precipitation variability and landslide occurrence in a subtropical mountain ecosystem of NW Argentina. Dendrochronologia 22: 175-180.

Paritsis, J., Veblen, T.T., Kitzberger, T. 2009. Assessing dendroecological methods to reconstruct defoliator outbreaks on Nothofagus pumilio in northwestern Patagonia, Argentina. Canadian Journal of Forest Research 39: 1617-1629.

Paritsis, J., Veblen, T.T. 2011. Dendroecological analysis of defoliator outbreaks on Nothofagus pumilio and their relation to climate variability in the Patagonian Andes. Global ChangeBiology 17: 239-253.

Pérez-de-Lis, G., García-González, I., Rozas, V., Arévalo, J.R. 2011. Effects of thinning intensity on radial growth patterns and temperature sensitivity in Pinus canariensis afforestations on Tenerife Island, Spain. Annals of Forest Science 68: 1093-1104.

Pollmann, W. 2002. Effects of natural disturbance and selective logging on Nothofagus forests in south-central Chile. Journal of Biogeography 29: 955-970.

Pollmann, W. 2003. Stand structure and dendroecology of an old-growth Nothofagus forest in Conguillio National Park, south Chile. Forest Ecology and Management 176:87-103.

Pompa-García, M., Domínguez-Calleros, P.A. 2015. Respuesta de madera temprana y tardía a la sequía en una conífera mexicana bajo dos condiciones ecológicas. Ecosistemas 24(2): 37-42.

Rebertus, A.J., Kitzberger, T., Veblen, T.T., Roovers, L.M. 1997. Blowdown history and landscape patterns in the Andes of Tierra del Fuego, Argentina. Ecology 78:678-692.

Rodriguez Catón, M., Villalba, R., Srur, A., Luckman, B.H. 2015. Long-term trends in radial growth associated with Nothofagus pumilio forest decline in Patagonia: Integrating local- into regional-scale patterns. Forest Ecology and Management 339: 44-56.

Rozas, V. 2003. Regeneration patterns, dendroecology, and forest-use history in an old-growth beech-oak lowland forest in Northern Spain. ForestEcology and Management 182: 175-194.

Rozas, V. 2004a. Análisis estructural y dendroecológico del roble (Quercus robur) en las carbayedas de Tragamón y la Isla, Gijón (Asturias). Ecología 18:127-143.

Rozas, V. 2004b. A dendroecological reconstruction of age structure and past management in an old-growth pollarded parkland in northern Spain. Forest Ecology and Management 195: 205-219.

Rozas, V. 2005. Regeneration patterns, dendroecology, and forest-use history in an old-growth beech-oak lowland forest in Northern Spain. Forest Ecology and Management 182: 175-194.

Rozas, V., Olano, J.M., DeSoto, L., Bartolomé, D. 2008. Large-scale structural variation and long-term growth dynamics of Juniperus thurifera trees in a managed woodland in Soria, central Spain. Annals of Forest Science 65: 809 .

Rozendaal, D.M., Soliz-Gamboa, C.C., Zuidema, P.A. 2011. Assessing longterm changes in tropical forest dynamics: a first test using tree-ring analysis. Trees 25: 115-124.

Sánchez-Salguero, R., Navarro-Cerillo, R.M., Camarero, J.J., FernándezCancio, A. 2010. Drought-induced growth decline of Aleppo and maritime pine forests in south-eastern Spain. Forest Systems 19: 458469.

Sangüesa-Barreda, G., Linares, J.C., Camarero, J.J. 2012. Mistletoe effects on Scots pine decline following drought events: insights from within-tree spatial patterns, growth and carbohydrates. TreePhysiology 32: 585-598.

Sangüesa-Barreda, G., Linares, J.C., Camarero, J.J. 2013. Drought and mistletoe reduce growth and water-use efficiency of Scots pine. Forest Ecology and Management 296: 64-73. 
Sangüesa-Barreda, G., Camarero, J.J., García-Martín, A., Hernández, R., de la Riva, J. 2014. Remote-sensing and tree-ring based characterization of forest defoliation and growth loss due to the Mediterranean pine processionary moth. Forest Ecology and Management 320:171-181.

Sangüesa-Barreda, G., Camarero, J.J., Oliva, J., Montes, F., Gazol, A. 2015a. Past logging, drought and pathogens interact and contribute to forest dieback. Agricultural and Forest Meteorology 208: 85-94.

Sangüesa-Barreda, G., Camarero, J.J., Linares, J.C., Hernández, R., Oliva, J., Gazol, A., et al. 2015b. Papel de los factores bióticos y las sequías en el decaimiento del bosque: aportaciones desde la dendroecología. Ecosistemas 24(2): 15-23.

Schöngart, J. 2008. Growth-Oriented Logging (GOL): A new concept towards sustainable forest management in Central Amazonian várzea floodplains. Forest Ecology and Management 256: 46-58.

Suarez, M.L., Kitzberger, T. 2010. Differential effects of climate variability on forest dynamics along a precipitation gradient in northern Patagonia. Journal of ecology 98:1023-1034.

Szejner, P., Le Quesne, C., González. M.E., Christie, D.A. 2011 Recuperación de bosques de Pilgerodendron uviferum (D. Don) Florin luego de perturbaciones antrópicas en la Cordillera de la Costa de Valdivia, Chile. Gayana Botánica 68: 196-206.

Tíscar, P.A. 2015. Modelo de ciclo forestal para la gestión sostenible de los bosques de Pinus nigra subsp. salzmannii deducido a partir de datos dendrocronológicos. Ecosistemas 24(2): 30-36.
Veblen, T. T., Kitzberger, T., Villalba, R., Donnegan, J. 1999. Fire history in northern Patagonia: the roles of humans and climatic variation. Ecological Monographs 69: 47-67.

Veblen, T.T., Kitzberger, T., Raffaele, E., Mermoz, M., González, M.E., Sibold, J.S., Holz, A. 2009. The historical range of variability of fires in the Andean-Patagonian Nothofagus forest region. International Journal of Wildland Fire 17:724-741.

Villagra, P.E., Villalba, R., Boninsegna, J. A. 2005a. Structure and growth rate of Prosopis flexuosa woodlands in two contrasting environments of the central Monte desert. Journal of Arid Environments 60: 187-199.

Villagra, P.E., Boninsegna, J. A., Alvarez, J.A., Cony, M., Cesca E., Villalba, R. 2005b. Dendroecology of Prosopis flexuosa woodlands in the Monte Desert: Implications for their management. Dendrochronologia 22: 209213.

Villalba R., Veblen T.T. 1997. Regional patterns of tree population age structures in northern Patagonia:climate and disturbance influences. Journal of Ecology 85: 113-124.

Voltas, J., Camarero, J.J., Carulla, D., Aguilera, M., Oriz, A. Ferrio, J.P. 2013. A retrospective, dual-isotope approach reveals individual predispositions to winter-drought induced tree dieback in the southernmost distribution limit of Scots pine. Plant, Cell and Environment 36: 1435-1448.

Worbes, M., Raschke, N. 2012. Carbon allocation in a Costa Rican dry forest derived from tree ring analysis. Dendrochronologia 30: 231-238 